\title{
1 Relationship between impulsivity, uncontrolled eating and body mass index: a
}

\section{2 hierarchical model}

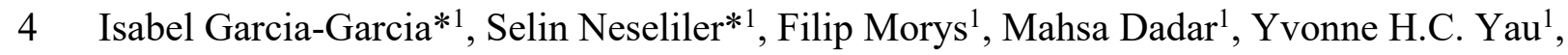

5 Stephanie G. Scala ${ }^{1}$, Yashar Zeighami ${ }^{1}$, Natalie Sun ${ }^{1}$, D. Louis Collins ${ }^{1}$, Uku Vainik ${ }^{1,2}$,

6 and Alain Dagher ${ }^{1}$

7

1. Montreal Neurological Institute, McGill University, Montréal, Canada

2. Institute of Psychology, University of Tartu, Estonia

10

$11 *$ Isabel García-García and Selin Neseliler should be considered first authors

12

14 Corresponding Author:

15 Alain Dagher MD,

16 Montreal Neurological Institute,

173801 University St., Montreal QC, Canada H3A 2B4

18 Tel: $514-398-1726$

19 Email: alain.dagher@mcgill.ca

22 Short/running title: Impulsivity and weight change

23 Keywords: Obesity, MRI, impulsivity, weight gain, Uncontrolled Eating 


\section{Abstract}

Background

26 Impulsivity increases the risk for obesity and weight gain. However, the precise role of impulsivity

27 in the aetiology of overeating behavior and obesity is currently unknown. Here we examined the

28 relationships between personality-related measures of impulsivity, Uncontrolled Eating, BMI, and

29 longitudinal weight changes. Additionally, we analyzed the associations between general

30 impulsivity domains and brain cortical thickness to elucidate brain vulnerability factors related to

31 weight gain.

32 Methods

33 Students in their first year of university - a risky period for weight gain - completed questionnaire

34 measures of impulsivity and eating behavior at the beginning $(\mathrm{N}=2318)$ of the school year. We

35 also collected their weight at the end of the term $(\mathrm{N}=1197)$. Impulsivity was divided into factors

36 stress reactivity, reward sensitivity and lack of self-control. Using structural equation models, we

37 tested the plausibility of a hierarchical relationship, in which impulsivity traits were associated

38 with Uncontrolled Eating, which in turn predicted BMI and weight change. 71 participants

39 underwent T1-weighted MRI to investigate the correlation between impulsivity and cortical

40 thickness.

41 Results

42 Impulsivity traits showed positive correlations with Uncontrolled Eating. Higher scores in

43 Uncontrolled Eating were in turn associated with higher BMI. None of the impulsivity-related

44 measurements nor Uncontrolled Eating were correlated with longitudinal weight gain. Higher

45 stress sensitivity was associated with increased cortical thickness in the superior temporal gyrus.

46 Lack of self-control was positively associated with increased thickness in the superior medial 
47 frontal gyrus. Finally, higher reward sensitivity was associated with lower thickness in the inferior

48 frontal gyrus.

49 Conclusion

50 The present study provides a comprehensive characterization of the relationships between different

51 facets of impulsivity and obesity. We show that differences in impulsivity domains might be

52 associated with BMI via Uncontrolled Eating. Our results might inform future clinical strategies

53 aimed at fostering self-control abilities to prevent and/or treat unhealthy weight gain.

54

55

56

57

58

59

60

61

62

63

64

65

66

67

68

69 


\section{$\underline{\text { Introduction }}$}

71 The increase in the incidence of obesity in the past 50 years can be attributed to overeating in

72 response to abundant and inexpensive calories (1). Obesity is also heritable, and most of the

73 implicated genes appear to be expressed in the central nervous system (2). As such, obesity can be

74 thought of as resulting from an interaction between a brain-based endophenotype and a disease-

75 promoting environment (3). Endophenotypes are intermediate phenotypes that link latent

76 biological processes to observable outcomes $(4,5)$. The neurobehavioral endophenotypes

77 associated with obesity can be broadly categorized as domain-general and eating-specific (6).

78 One domain-general endophenotype is impulsivity (7), the tendency to act without full

79 consideration of the consequences (8). It can be subdivided into three domains that align with

80 personality factors: 1) low conscientiousness, reflecting self-control, or, in other terms,

81 premeditation and perseverance ; 2) neuroticism, a reflection of an individual's sensitivity to stress

82 and aversive events, also referred to as negative urgency, or the tendency to act impulsively when

83 distressed, and 3) extraversion, reflecting sensitivity to rewards and sensation seeking (8-10).

84 Enhanced impulsivity is characteristic of different neuropsychiatric conditions, such as

85 pathological gambling, substance abuse and attention deficit hyperactivity disorder $(11,12)$.

86 Additionally, it might constitute a risk factor for obesity and overeating patterns (13-15).

87 Meta-analytic studies have shown that impulsivity shows weak positive correlations with BMI

88 across independent studies (16). The effects of impulsivity on BMI, however, seem to be largely

89 heterogeneous (16). One of the reasons for this heterogeneity could be explained by different

90 impulsivity measures and domains used across studies (6). Another possibility is that the relation

91 between personality measures of impulsivity and BMI might be mediated by individually-varying

92 eating-specific impulsivity characteristics (17). In terms of brain correlates of impulsivity, it seems 
93 that they overlap with brain correlates of high BMI, which suggests that there is a relationship

94 between obesity and impulsivity that might stem from the brain. For example, both high

95 impulsivity and BMI are positively related to the volume of the striatum $(18,19)$, or negatively

96 related to the volume of the prefrontal and orbitofrontal cortex $(20,21)$.

98 Eating-specific constructs associated with a high BMI include emotional eating (22), disinhibited 99 eating (23), and power of food (24). Scores on all these questionnaires consistently and strongly 100 correlate with BMI (25) and with each other (26). For this reason, in previous research we have 101 proposed that different eating behavior questionnaires depict a common underlying latent factor, 102 labelled uncontrolled eating $(25,27)$. Preliminary results show that uncontrolled eating is 103 associated with brain alterations from functional MRI studies, specifically higher brain activity in 104 response to food cues or at rest in the cerebellum, and lower brain activity in the prefrontal cortex 105 (28). While both impulsivity traits and uncontrolled eating have been linked to increased BMI 106 (17), the relationships between different impulsivity domains, uncontrolled eating and obesity 107 remain to be tested. A hypothesis here is that some impulsivity traits might be associated with 108 eating behaviors, which in turn will be linked to BMI (17). However, comprehensive models have 109 not been built for different impulsivity domains and eating questionnaires and have not been 110 applied to the prediction of weight changes yet.

111 So far, most analyses have been conducted on cross-sectional datasets making it difficult to say if 112 impulsivity is a risk factor or a consequence of obesity (28). The freshman year of university is an 113 ideal time period to test these hypotheses both cross-sectionally and longitudinally. During this 114 time, students transition into a new environment with access to similar food and exercise options, 115 allowing underlying vulnerability to express itself. Weight gain often happens in this short period 
116 of time and affects approximately $50-60 \%$ of students $(29,30)$. Eating specific behaviors that

117 revolve around the concept of impulsivity have been most commonly studied as risk factors for

118 weight gain. The results, however, have been contradictory (31-33).

119 In the brain, a high BMI has been associated with lower gray matter volume and cortical thickness

120 in areas such as the medial prefrontal cortex extending to the orbitofrontal cortex, and temporal

121 pole $(21,34,35)$. However, it is possible that other brain structures involved in processes like

122 impulsivity might be indirectly associated with weight gain. Identifying those regions might pave

123 the way for new studies to investigate links between brain function and maladaptive eating

124 patterns.

125 In the current study, we present a comprehensive examination of the relationships between

126 impulsivity, uncontrolled eating, BMI and weight changes. We tested the hypothesis that the

127 relations between general impulsivity, uncontrolled eating, BMI and longitudinal weight changes

128 might be plausibly represented in a hierarchical structural equation model (SEM). In a sub-sample

129 of participants, we additionally examined the relationship between domain-general impulsivity

130 variables and brain structure. This post-hoc analysis was performed in order to extend our

131 behavioral findings and identify new brain structures that might be relevant to weight status in an

132 indirect manner.

134 Methods and Materials

135 Participants

136 Participants were first year McGill university students, at least 18 years of age, recruited via an

137 advertisement sent to the incoming class electronic mailing list. Participants provided their consent

138 online and data were collected using the online survey tool LimeSurvey 
139 (https://www.limesurvey.org) over three consecutive years. The study was approved by the

140 Montreal Neurological Institute Research Ethics Board. Participants for the brain imaging

141 experiment gave additional written consent before participating.

143 Questionnaires

144 Participants filled out the following online questionnaires in the fall semester: "Big Five" 145 personality dimensions (Openness/ Imagination, Conscientiousness, Agreeableness, Extraversion, 146 and Neuroticism) from the International Personality Item Pool (IPIP) (36), two subscales (lack of 147 perseverance and sensation seeking) of the UPPS Impulsive Behavior Scale (37), Cohen's 148 Perceived Stress Scale (PSS) (38), and Rosenberg Self Esteem Scale (39), as well as eating specific 149 questionnaires such as the disinhibition subscale of Three-Factor Eating Questionnaire (TFEQ) 150 (23), emotional eating subscale of Dutch Eating Behavior Questionnaires (DEBQ) (22), and all 151 subscales of Power of Food Scale (PFS) (24) (Table 1). Participants also reported their height and 152 weight at two timepoints - together with online questionnaires during the initial assessment in the 153 fall semester, and during a follow-up in the spring semester. Only a subset of participants reported 154 their height and weight during the follow-up (N=1145). Our questionnaires included two "catch" 155 questions and three "catch-match" questions as a measure of the level of participants' attention in 156 completing the survey. Participants with total catch scores above three were excluded, resulting in 157 a sample of 2318 participants (Males $\mathrm{N}=750$; Females $\mathrm{N}=1538$ ). After completion of the 158 questionnaires during the initial assessment and after the follow-up, a subset of participants were 159 asked to visit our laboratory to have their BMI measured. Height and weight were measured from 160333 participants in the fall, and from 209 participants in the spring semester $(\mathrm{N}=115$ overlap with 161 the fall group) using a medical scale and a stadiometer. Self-reported BMI was highly correlated 
162 with measured BMI in the fall $\left(\mathrm{r}=0.91, \mathrm{p}<10^{-5}\right)$ and in the spring $\left(\mathrm{r}=0.92, \mathrm{p}<10^{-5}\right)$. We replaced

163 the reported BMI with measured BMI in the analyses for higher accuracy. BMI was further

164 residualized for age, sex and a covariate to account for whether it was derived from self-report or

165 measured. Brain imaging was conducted on a subset of participants selected from this sample in

166 the fall $(\mathrm{N}=71$ participants, $\mathrm{N}=69$ of them were included in the cortical thickness analysis, see

167 below).

169 Structural Equation Models

170 We performed a series of SEMs to analyze the relationships between Uncontrolled Eating, the

171 three impulsivity traits defined above, and weight gain. First, we tested the hypothesis that

172 Uncontrolled Eating, stress reactivity, lack of self-control and reward sensitivity can be considered

173 separable latent constructs. We built a four-dimension model (Model 1, Figure 1A), with

174 Uncontrolled Eating, stress reactivity, lack of self-control and reward sensitivity modelled as latent

175 constructs. Uncontrolled Eating (UE) was defined by the scores in Disinhibition, Power of Food

176 and Emotional Eating, following previous work from our group $(27,28)$. Stress reactivity was the

177 latent variable that resulted from Perceived Stress Scale (PSS), Rosenberg self-esteem

178 questionnaire and Neuroticism (IPIP). Lack of Self Control was a latent factor emerging from the

179 observable variables Lack of Perseverance (UPPS) and Conscientiousness (IPIP). Finally, reward

180 Sensitivity was formed by Extraversion (IPIP) and Sensation Seeking (UPPS). This model was

181 compared to a simpler one-dimension model (Model 2, Figure 1B), where all the observable

182 factors were fit into a single latent variable.

183 We also built a hierarchical version of the four-dimension model (Model 3, Figure 1C), where we 184 examined the plausibility of a layered model structure, inspired by the watershed model of mental 
185 illness endophenotypes $(5,40)$. In this model, stress reactivity, lack of self-control, and reward

186 sensitivity were assumed to each contribute to the Uncontrolled Eating phenotype.

187 Finally, we examined the relationships between Uncontrolled Eating, the impulsivity traits, BMI

188 and longitudinal changes in BMI in a hierarchical four-dimension model (Model 4, Figure 1D).

189 We tested whether BMI and longitudinal change in BMI (delta BMI) were predicted by

190 Uncontrolled Eating, stress reactivity, lack of self-control and reward sensitivity.

191 We used the Lavaan package in R version 3.3. (41) to perform SEM. Model fit was assessed with

192 the chi-square test $\left(\mathrm{X}^{2}\right)$, the Comparative Fit index (CFI), Root Mean Square Error of 193 Approximation (RMSEA) and the standardized root mean squared residuals (SRMR). The

194 following guidelines were utilized for judging good fit: RMSEA (acceptable fit $<0.08$, good fit $<$ 195 0.05), CFI (acceptable fit 0.95-0.97, good fit >0.97), SRMR (acceptable fit 0.05-0.10, good fit $<$ $1960.05)(42)$.

197 To test for the robustness of the preferred hierarchical SEM model (model 3, Figure 1C), we 198 repeated the analysis by randomly splitting the sample in half. Age and sex were regressed out of 199 all the observable variables in the models. To account for the multiple models performed, we set 200 a stringent $p$ value threshold $(\mathrm{p}<0.005)$

202 Magnetic Resonance Imaging parameters and preprocessing

203 High-resolution T1-weighted anatomical images with voxel size $=1 \times 1 \times 1 \mathrm{~mm}$ were obtained $(\mathrm{TR}$ $204=2.3 \mathrm{~s} ; \mathrm{TE}=2.98 \mathrm{~ms} ; \mathrm{FOV}$ phase=93.8 $; \mathrm{FOV}=256 \mathrm{~mm}$ ) with a Siemens Magnetom Trio 3T 205 MRI scanner at the Montreal Neurological Institute (MNI). 
207 Pre-processing of T1-weighted MRIs included denoising using optimized non-local means

208 filtering (43), correction for intensity inhomogeneity (44) and linear intensity scaling using

209 histogram matching to the ICBM-MNI152 template. The images were linearly registered to the

210 ICBM-MNI152 template (9 parameter registration) (45). A mask of the brain was generated using

211 BEaST, a nonlocal segmentation method applied to the linearly registered images in stereotaxic

212 space (46).

\section{Cortical Thickness}

215 All T1-weighted MRI images were processed using the CIVET pipeline (version 2.1;

216 http://www.bic.mni.mcgill.ca/ServicesSoftware/CIVET). Native T1-weighted MRI scans were

217 first corrected using the N3 algorithm, underwent brain masking, and registration to ICBM-

218 MNI152 template. Images were then segmented into gray and white matter, cerebrospinal fluid

219 and background with a neural net classifier. The white matter (inner) and gray matter (outer)

220 cortical surfaces were generated using the Constrained Laplacian-based Automated Segmentation

221 with Proximities algorithm. These surfaces were resampled to a stereotaxic surface template to

222 allow vertex-based measurement of cortical thickness. All resulting images went through a

223 stringent quality control by two inspectors in which 69 of 71 images were accepted for further

224 analysis. Cortical thickness in MNI space was defined as the linked distance between the two

225 surfaces across vertices.

227 Post-hoc brain-impulsivity analysis

228 We performed a post-hoc exploratory analysis to test which brain regions are associated with the

229 three general-impulsivity domains. We extracted the latent individual scores for stress reactivity, 
230 lack of self-control and reward sensitivity of the participants with an available MRI. Next, using

231 the SurfStat software package (http://www.math.mcgill.ca/keith/surfstat/), we computed three

232 separate linear regression models to test for the associations between the three latent impulsivity

233 scores and cortical thickness. The models were analyzed with random field theory with a threshold

234 of $\mathrm{p}<0.05$ corrected for multiple comparisons over the entire surface (47). Results are reported

235 cluster- and vertex-wise corrected. Total surface area was included as covariate. Age and sex were

236 already regressed out of the observable variables forming the latent scores (see Structural Equation

237 Models above). For this reason, these confounding variables were not included in the analyses.

238 Moreover, since the latent scores were obtained from a SEM model that includes the 3 general

239 impulsivity variables as latent variables, each latent score "accounts" for the other two.

$241 \quad \underline{\text { Results }}$

242 Participants' characteristics are listed in Table 1.

Uncontrolled Eating and general impulsivity traits can be considered independent latent

245 variables (Models 1 and 2)

246 A four-dimension model, with Uncontrolled Eating, stress reactivity, lack of self-control and

247 reward sensitivity as latent variables provided an acceptable fit $\left(\chi_{(29)}^{2}=322.40 ;\right.$ RMSEA $=0.068$;

$248 \mathrm{CFI}=0.956 ;$ SRMR $=0.037)$. The first latent factor, Uncontrolled Eating, had positive loadings

249 from Disinhibited Eating, Power of Food, and Emotional Eating. The latent factor stress reactivity

250 had a negative loading from the Rosenberg self-esteem questionnaire and positive loadings from

251 Neuroticism and the Perceived Stress Scale. Lack of self-control, the third latent factor had a

252 positive loading from Lack of Perseverance and a negative loading from Conscientiousness.

253 Reward sensitivity was characterized by positive loadings from the Extraversion and Sensation 
254 Seeking (UPPS) subscales. This model provided a better fit than an alternative single-dimension 255 model, where all the observable variables were fit into a single latent score (difference in $\chi^{2}{ }_{(6)}=$ $256 \quad 2770.5 ; \mathrm{p}=2.2 \mathrm{e}-16)$.

A four-dimensional hierarchical model, where general impulsivity traits predict

Uncontrolled Eating, is plausible (Model 3)

260 We tested the plausibility of a four-dimension hierarchical model. In this model, the latent

261 variables stress reactivity, lack of self-control and reward sensitivity were characterized as

262 predictors of Uncontrolled Eating. All the paths were significant. Note that the fit parameters are

263 the same as Model 2, since sample size and degrees of freedom did not change and provided an

264 acceptable fit (Figure 2A).

Relationship between Uncontrolled Eating, BMI and longitudinal weight change (Model 4)

267 Finally, we tested the relationship between Uncontrolled Eating, general impulsivity, BMI and

268 longitudinal changes in BMI in the hierarchical four-dimension model. From these additional

269 regression paths, the only one that was significant was the association between BMI and

270 Uncontrolled Eating $(\mathrm{B}=0.38 ; \mathrm{SE}=0.05 ; \mathrm{Beta}=0.09 ; \mathrm{p}<0.001)$. This model provided an acceptable

271 fit $\left(\chi^{2}(41)=198.52 ; \mathrm{RMSEA}=0.055 ; \mathrm{CFI}=0.956 ; \mathrm{SRMR}=0.032\right)($ Figure $2 \mathrm{~B})$.

\section{Validation of Model 4 in the half split dataset}

274 All the previously observed relationships in Model 4 remained equivalent in the two subsamples.

275 Fit parameters in the first half of the sample $(\mathrm{N}=601$ observation $)$ were as follows: $\left(\chi^{2}(41)=102.97\right.$;

276 RMSEA $=0.050 ; \mathrm{CFI}=0.965 ; \mathrm{SRMR}=0.032$ ). The same model in the second half of the sample 
$277(\mathrm{~N}=600$ observations $)$ had the following fit parameters $\left(\chi^{2}(41)=142.72\right.$; RMSEA $=0.064$; CFI $=$

278 0.939; $\mathrm{SRMR}=0.039)$.

\section{Post-hoc correlations between cortical thickness and general impulsivity scores}

281 Higher stress sensitivity was associated with greater cortical thickness in the right temporoparietal

282 junction extending to superior temporal gyrus. Lack of self-control was positively associated with

283 greater thickness in the right superior frontal gyrus extending to midcingulate cortex. Finally,

284 higher scores in reward sensitivity were associated with lower thickness in the bilateral inferior 285 frontal gyrus (Figure 3).

\section{Discussion}

288 The purpose of the current study was to provide a comprehensive examination of the relationship

289 between domain-general (personality-related facets of impulsivity) and eating-specific obesity

290 endophenotypes (Uncontrolled Eating), BMI, and weight changes. To do so, we tested a series of

291 structural equation models in a large sample size $(\mathrm{N}>2300)$ of first-year students and accounted

292 for the multidimensional nature of impulsivity. A final SEM model, which depicted a hierarchical

293 relationship between general impulsivity, Uncontrolled Eating, BMI and weight gain, was

294 developed in the first split-half of the sample and replicated in the second. In a follow-up analysis

295 we also investigated neural correlates of different facets of impulsivity. We found that cortical

296 thickness in parts of the frontal, cingulate and temporoparietal cortex were correlated with

297 impulsivity and hence are potentially related to BMI via Uncontrolled Eating. 
299 Following previous work, we first stratified impulsivity into three general latent variables: stress

300 sensitivity, lack of self-control and reward sensitivity $(8,10)$. A fourth latent variable, Uncontrolled

301 Eating, was considered an eating-specific form of impulsivity. In line with previous publications

302 (27), Uncontrolled Eating was defined using the total scores of relevant eating questionnaires. We

303 showed that an SEM that separated these 4 latent factors was significantly better than a model in

304 which all the observable variables were loaded into a single latent score. Moreover, a hierarchical

305 relationship, in which the three general impulsivity domains were considered predictors of

306 Uncontrolled Eating, was found to be plausible. Each general impulsivity domain had an

307 independent and positive correlation with Uncontrolled Eating. We also found that higher scores

308 in Uncontrolled Eating were positively associated with BMI, as demonstrated previously $(27,28)$.

309 However, none of the relations between general impulsivity domains and BMI were significant in

310 the model that also included Uncontrolled Eating. The SEM model therefore suggests that the

311 association between impulsivity and BMI is indirect, with impulsivity factors predicting

312 Uncontrolled Eating, and Uncontrolled Eating predicting BMI.

314 Finally, we tested the hypothesis that Uncontrolled Eating underlies vulnerability for weight gain

315 during the first year of university. We utilized the transition period into university because it is

316 associated with a high risk of weight gain. In our sample, the average weight gain was small (0.52

$317 \mathrm{~kg}$ over 10 months), but significant, and was similar to previous reports (29). However, in this

318 sample we did not find evidence that Uncontrolled Eating can longitudinally predict weight gain.

319 This could be due to limited duration of the study. Moreover, we cannot exclude the possibility of

320 seasonal weight gain (48), which would not necessarily be related to Uncontrolled Eating. The

321 existence of bidirectional relationships between Uncontrolled Eating and BMI might also mask a 
relationship, since changes in body weight might lead to longitudinal variations in eating-specific

323 impulsivity (49). For instance, scores in different Uncontrolled Eating scales decrease after

324 bariatric surgery (50) or voluntary weight loss (51).

326 Our results support a model in which BMI is associated with Uncontrolled Eating, which in turn

327 is derived from general impulsivity. Impulsivity could compromise the maintenance of behaviors

328 that promote healthy weight. High scores on conscientiousness have been consistently linked to a

329 lower risk of having and developing obesity across independent samples $(52,53)$. These results are

330 generally derived from sample populations with a higher mean age than ours, suggesting that self-

331 control deficits may be more associated with BMI as individuals age and start making their own

332 food decision. Nonetheless, our findings point to reduced conscientiousness (lack of self-control)

333 as a risk factor for overeating behaviors in adolescent and young adult populations.

334 In a subset of the sample, we performed additional post-hoc correlations between cortical thickness

335 and impulsivity in all three tested domains. We found that the temporoparietal junction, superior

336 frontal gyrus/midcingulate cortex and inferior frontal gyrus were associated with stress sensitivity,

337 lack of self-control and reward sensitivity, respectively. These results are consistent with previous

338 reports showing brain correlates of impulsivity $(19,20,54)$ and partially overlap with obesity-

339 related brain regions $(21,55)$. Paralleled by our SEM results, these findings show that cortical

340 thickness differences might indirectly influence BMI via Uncontrolled Eating. In the future we

341 would like to update our SEM model to test the hypothesis that brain differences can act as

342 independent predictors of impulsivity, which in turn are significant predictors of Uncontrolled

343 Eating, and hence BMI. This hierarchy of relationships can be depicted as a watershed model (40),

344 which will allow to test the hypothesis that the temporoparietal junction, midcingulate cortex and 
345 inferior frontal gyrus are indirectly involved in BMI, via their association with impulsivity.

346 Unfortunately, due to low imaging sample size, this was not possible in this study.

348 The results of our study should be considered with regards to its limitations. Although we had a

349 large sample group, whereby we were able to detect and replicate results from meta-analyses, most

350 of our measures were self-reported. In addition, brain imaging was conducted with a small subset

351 of the total sample.

Acknowledgments

354 This research was supported by a Canadian Institutes of Health Research Grant to AD. IGG was

355 the recipient of a Postdoctoral Fellowship from the Canadian Institutes of Health Research. SN

356 was supported by a Frederick Banting and Charles Best Canada Graduate Scholarship. UV was

357 supported by Personal Post-doctoral Research Funding project PUTJD654 and by Fonds de 358 recherche du Québec - Santé (FRQS) foreign post-doctoral training award.

\section{Competing interests}

361 IGG, SN, FM, MD, YY, SGS, YZ, NS, DLC, UV, AD report no financial interests or potential 362 conflicts of interest.

\section{$364 \underline{\text { References }}$}

365 1. Hall KD. Did the Food Environment Cause the Obesity Epidemic? Obesity [Internet].

3662018 Jan 1 [cited 2020 Aug 25];26(1):11-3. Available from:

$367 \quad /$ pmc/articles/PMC5769871/?report=abstract

368 2. Locke AE, Kahali B, Berndt SI, Justice AE, Pers TH, Day FR, et al. Genetic studies of 
body mass index yield new insights for obesity biology. Nature [Internet]. 2015 Feb 12

/pmc/articles/PMC4382211/?report=abstract

372 3. O'Rahilly S, Farooqi IS. Human obesity: a heritable neurobehavioral disorder that is highly sensitive to environmental conditions. Diabetes. 2008 Nov;57(11):2905 LP - 2910.

374 4. Dagher A, Neseliler S, Han J-E. Chapter 32 - Appetite as Motivated Choice: Hormonal and Environmental Influences. In: Dreher J-C, Tremblay LBT-DN, editors. Decision

5. Cannon TD, Keller MC. Endophenotypes in the Genetic Analyses of Mental Disorders. Annu Rev Clin Psychol. 2006 Mar;2(1):267-90.

379 6. Vainik U, Dagher A, Dubé L, Fellows LK. Neurobehavioural correlates of body mass index and eating behaviours in adults: A systematic review. Neurosci Biobehav Rev.

382 7. Michaud A, Vainik U, Garcia-Garcia I, Dagher A. Overlapping Neural Endophenotypes in Addiction and Obesity. Front Endocrinol (Lausanne) [Internet]. 2017 Jun 14 [cited 2019 Sep 3];8:127. Available from:

386 8. DeYoung CG. Impulsivity as a personality trait. In: Handbook of self-regulation: 387 Research, theory, and applications, 2nd ed. New York, NY, US: Guilford Press; 2011. p. $388 \quad 485-502$.

389 9. Settles RE, Fischer S, Cyders MA, Combs JL, Gunn RL, Smith GT. Negative urgency: a personality predictor of externalizing behavior characterized by neuroticism, low 
2020 Aug 25];121(1):160-72. Available from:

/pmc/articles/PMC3299541/?report=abstract

394 10. Sharma L, Markon KE, Clark LA. Toward a theory of distinct types of "impulsive"

395 behaviors: A meta-analysis of self-report and behavioral measures. Psychol Bull. 2014

$396 \quad$ Mar;140(2):374-408.

397 11. Dalley JW, Robbins TW. Fractionating impulsivity: neuropsychiatric implications. Nat $398 \quad$ Rev Neurosci. 2017;18(3):158-71.

399 12. Brooks SJ, Lochner C, Shoptaw S, Stein DJ. Using the research domain criteria (RDoC) to conceptualize impulsivity and compulsivity in relation to addiction. 1st ed. Vol. 235, Progress in Brain Research. Elsevier B.V.; 2017. 177-218 p.

402 13. Manasse SM, Espel HM, Schumacher LM, Kerrigan SG, Zhang F, Forman EM, et al. 403 Does impulsivity predict outcome in treatment for binge eating disorder? A multimodal 404 investigation. Appetite. 2016;105:172-9.

405 14. Meule A, Blechert J. Interactive and indirect effects of trait impulsivity facets on body 406 mass index. Appetite. 2017;118:60-5.

407 15. Kishinevsky FI, Cox JE, Murdaugh DL, Stoeckel LE, Cook III EW, Weller RE. fMRI reactivity on a delay discounting task predicts weight gain in obese women. Appetite. 2012;58(2):582-92.

410 16. Emery RL, Levine MD. Questionnaire and behavioral task measures of impulsivity are 411 differentially associated with body mass index: A comprehensive meta-analysis. Psychol $412 \quad$ Bull. 2017;143(8):868-902.

413 17. Meule A, Blechert J. Indirect effects of trait impulsivity on body mass. Eat Behav. 2017 Aug;26(Supplement C):66-9. 
415 18. García-García I, Morys F, Dagher A. Nucleus accumbens volume is related to obesity measures in an age-dependent fashion. J Neuroendocrinol [Internet]. 2019 Nov 23 [cited 2019 Dec 2]; Available from: https://onlinelibrary.wiley.com/doi/abs/10.1111/jne.12812

418 19. Tschernegg M, Pletzer B, Schwartenbeck P, Ludersdorfer P, Hoffmann U, Kronbichler M. Impulsivity relates to striatal gray matter volumes in humans: evidence from a delay discounting paradigm. Front Hum Neurosci [Internet]. 2015 Jul 2 [cited 2020 Jun 9];9(JULY):384. Available from: http://journal.frontiersin.org/Article/10.3389/fnhum.2015.00384/abstract

20. Matsuo K, Nicoletti M, Nemoto K, Hatch JP, Peluso MAM, Nery FG, et al. A voxel-based morphometry study of frontal gray matter correlates of impulsivity. Hum Brain Mapp. 2009 Apr;30(4):1188-95.

21. García-García I, Michaud A, Dadar M, Zeighami Y, Neseliler S, Collins DL, et al.

429 22. van Strien T, Frijters JER, Bergers GPA, Defares PB. The Dutch Eating Behavior Questionnaire (DEBQ) for assessment of restrained, emotional, and external eating behavior. Int J Eat Disord. 1986 Feb;5:295-315.

432 23. Stunkard AJ, Messick S. The Three-Factor Eating Questionnaire to measure dietary restraint, disinhibition and hunger. J Psychosom Res. 1985;29(1):71-83.

434 24. Lowe MR, Butryn ML, Didie ER, Annunziato RA, Thomas JG, Crerand CE, et al. The Power of Food Scale. A new measure of the psychological influence of the food environment. Appetite. 2009;53(1):114-118.

437 25. Price M, Higgs S, Lee M. Self-reported eating traits: Underlying components of food 
responsivity and dietary restriction are positively related to BMI. Appetite. 2015;95:203-

10.

440 26. Mason AE, Vainik U, Acree M, Tomiyama AJ, Dagher A, Epel ES, et al. Improving assessment of the spectrum of reward-related eating: The RED-13. Front Psychol. 2017;8(MAY).

443 27. Vainik U, Neseliler S, Konstabel K, Fellows LK, Dagher A. Eating traits questionnaires as a continuum of a single concept. Uncontrolled eating. Appetite. 2015;

445 28. Vainik U, García-García I, Dagher A. Uncontrolled eating: a unifying heritable trait linked with obesity, overeating, personality and the brain. Eur J Neurosci. 2019;50.

29. Vadeboncoeur C, Townsend N, Foster C. A meta-analysis of weight gain in first year university students: is freshman 15 a myth? BMC Obes. 2015;2(1):22.

449 30. Provencher V, Polivy J, Wintre MG, Pratt MW, Pancer SM, Birnie-Lefcovitch S, et al.

$450 \quad$ Who gains or who loses weight? Psychosocial factors among first-year university students. Physiol Behav. 2009;96(1):135-41.

452 31. Finlayson G, Cecil J, Higgs S, Hill A, Hetherington M. Susceptibility to weight gain. Eating behaviour traits and physical activity as predictors of weight gain during the first year of university. Appetite. 2012 Jun;58(3):1091-8.

455 32. Lowe MR, Annunziato RA, Markowitz JT, Didie E, Bellace DL, Riddell L, et al. Multiple 456 types of dieting prospectively predict weight gain during the freshman year of college. $457 \quad$ Appetite. 2006;47(1):83-90.

458 33. Meule A, Platte P. An examination of the "Freshman-15" in Germany: Predictors of weight change in female university students. Vol. 25, European Journal of Health 
Hellbrunner Strasse 34, Salzburg, Austria, 5020, adrian.meule@sbg.ac.at: Hogrefe Publishing; 2018. p. 2-8.

463 34. Beyer F, Kharabian Masouleh S, Kratzsch J, Schroeter ML, Röhr S, Riedel-Heller SG, et al. A metabolic obesity profile is associated with decreased gray matter volume in cognitively healthy older adults. Front Aging Neurosci. 2019;11(August):1-14.

35. Medic N, Ziauddeen H, Ersche KD, Farooqi IS, Bullmore ET, Nathan PJ, et al. Increased body mass index is associated with specific regional alterations in brain structure. Int $\mathrm{J}$ Obes. 2016 Jul 1;40(7):1177-82.

36. Donnellan MB, Oswald FL, Baird BM, Lucas RE. The Mini-IPIP Scales: Tiny-yeteffective measures of the Big Five Factors of Personality. Vol. 18, Psychological Assessment. Donnellan, M. Brent: Department of Psychology, Michigan State University, East Lansing, MI, US, 48823, donne159@msu.edu: American Psychological Association; 2006. p. 192-203.

38. Cohen S, Kamarck T, Mermelstein R. A global measure of perceived stress. J Health Soc Behav. 1983;24(4):385-96.

478 39. Rosenberg M. Society and the adolescent self-image. 1965.

479 40. Fuhrmann D, Simpson-Kent IL, Bathelt J, Holmes J, Gathercole S, Astle D, et al. A 480 Hierarchical Watershed Model of Fluid Intelligence in Childhood and Adolescence. Cereb Cortex. 2019;(June):1-14.

482 41. Rosseel Y. lavaan: An R Package for Structural Equation Modelinge human forearm during rythmic exercise. J Stat Softw. 2012;48(2):1-36. 
484 42. Schermelleh-Engel K, Moosbrugger H, Müller H. Evaluating the fit of structural equation models: Tests of significance and descriptive goodness-of-fit measures. MPR-online. 2003;8(May):23-74.

43. Coupe P, Yger P, Prima S, Hellier P, Kervrann C, Barillot C. An optimized blockwise nonlocal means denoising filter for 3-D magnetic resonance images. IEEE Trans Med Imaging. 2008;27(4):425-41.

44. Sled JG, Zijdenbos a P, Evans a C. A nonparametric method for automatic correction of

45. Collins DL, Neelin P, Peters TM, Evans AC. Automatic 3D intersuject registration for MR volumetric data in standardized Talairach space. Vol. 18, Journal of Computer Assisted Tomography. 1994. p. 192-205.

46. Eskildsen SF, Coupé P, Fonov V, Manjón J V, Leung KK, Guizard N, et al. BEaST: Brain extraction based on nonlocal segmentation technique. Neuroimage. 2012;59(3):2362-73.

47. Worsley KJ, Taylor JE, Tomaiuolo F, Lerch J. Unified univariate and multivariate random

48. Yanovski JA, Yanovski SZ, Sovik KN, Nguyen TT, O’Neil PM, Sebring NG. A 2020 Aug 24];342(12):861-7. Available from: analyses to elucidate causality in the associations between body mass index and psychological traits. 2020 [cited 2020 Aug 24]; Available from: 
507 50. Holsen LM, Davidson P, Cerit H, Hye T, Moondra P, Haimovici F, et al. Neural predictors of 12-month weight loss outcomes following bariatric surgery. Int J Obes. 2017;(August):1-9.

510 51. Batra P, Das SK, Salinardi T, Robinson L, Saltzman E, Scott T, et al. Relationship of cravings with weight loss and hunger. Results from a 6month worksite weight loss intervention. Appetite. 2013;69:1-7.

513 52. Jokela M, Hintsanen M, Hakulinen C, Batty GD, Nabi H, Singh-Manoux a., et al. Association of personality with the development and persistence of obesity: a metaanalysis based on individual-participant data. Obes Rev. 2012 Nov;14(4):315-23.

516 53. Terracciano A, Sutin AR, McCrae RR, Deiana B, Ferrucci L, Schlessinger D, et al. Facets of personality linked to underweight and overweight. Psychosom Med. 2009 Jul;71(6):682-9.

519 54. Cho SS, Pellecchia G, Aminian K, Ray N, Segura B, Obeso I, et al. Morphometric correlation of impulsivity in medial prefrontal cortex. Brain Topogr. 2013 Jul;26(3):479_ 87.

522 55. Vainik U, Baker TE, Dadar M, Zeighami Y, Michaud A, Zhang Y, et al. Neurobehavioral correlates of obesity are largely heritable. Proc Natl Acad Sci. 2018 Sep;115(37):9312-7. 


\section{Figures}

A
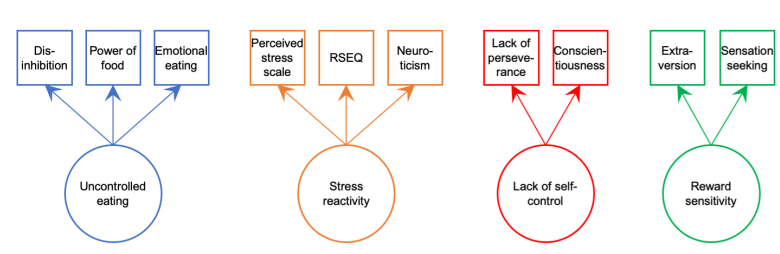

C

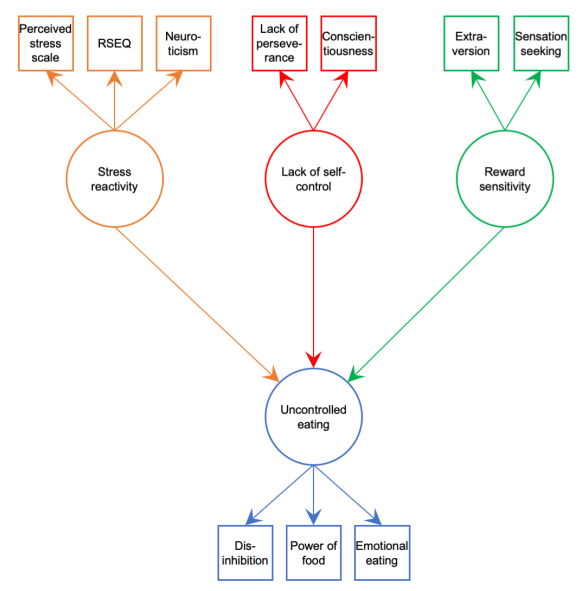

B

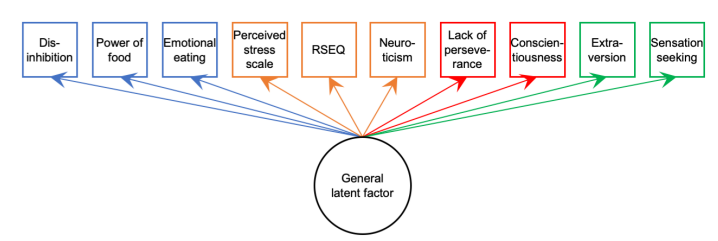

D

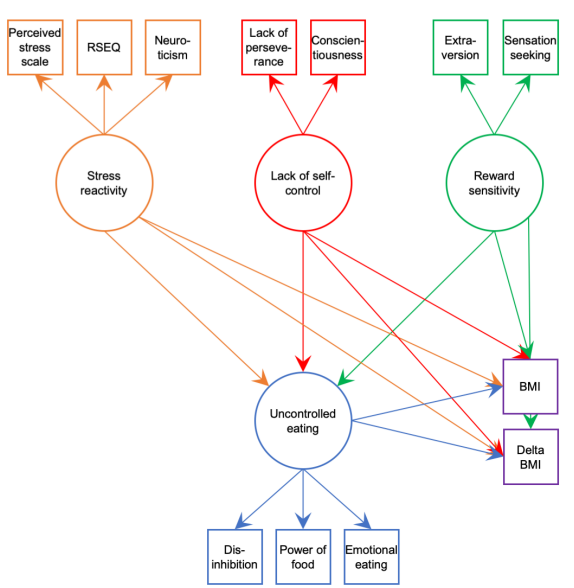

528 Figure 1 Structural equation models used in the study. A Model 1 representing 4 distinct latent

529 variables; B Model 2 pooling all measures into 1 latent variable; C Model 3 representing a

530 hierarchical model where stress reactivity, lack of self-control and reward sensitivity all

531 influence Uncontrolled Eating; D Model 4 where all 4 latent variables influence BMI and BMI

532 change. RSEQ - Rosenberg self-esteem questionnaire. BMI - body mass index. 
A

533

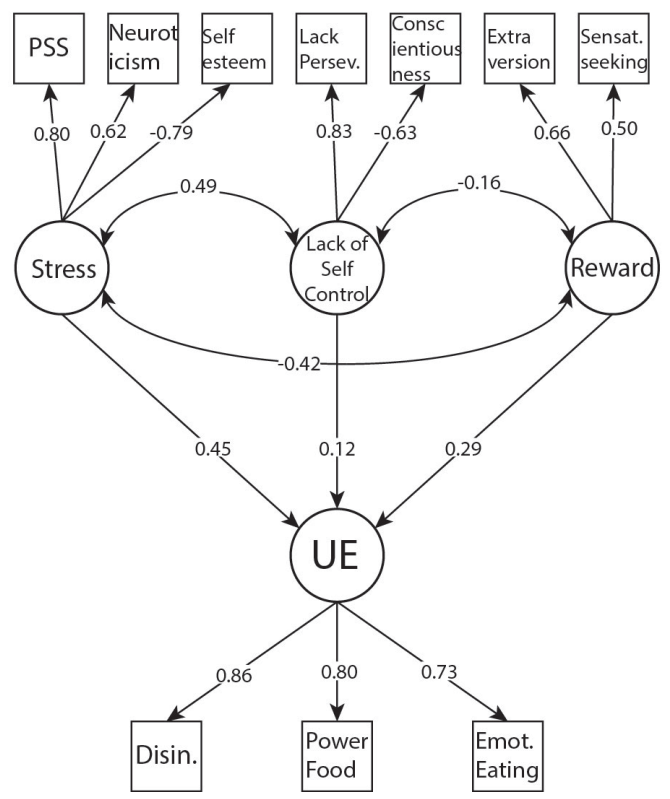

B

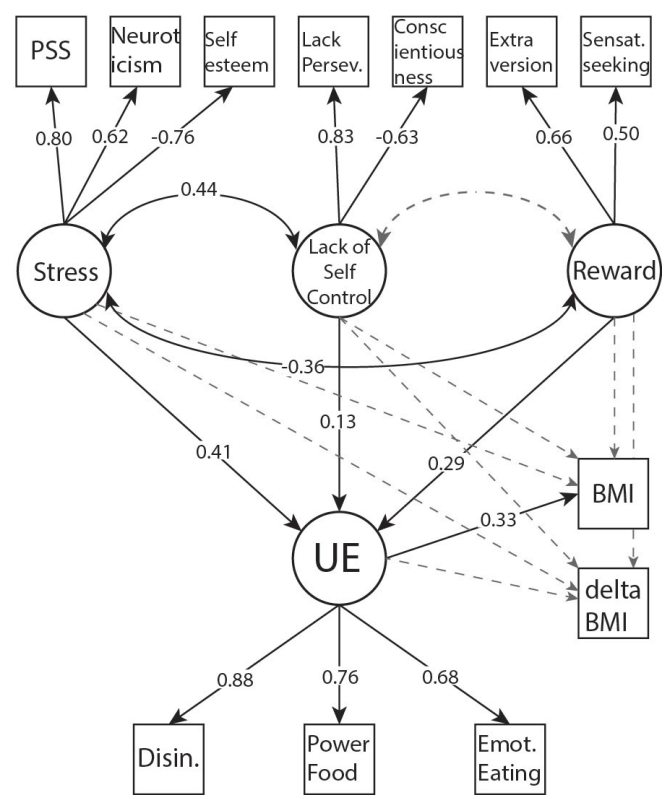

534 Figure 2. SEM representing: A) Model $3(\mathrm{n}=2318)$, the relationships between Uncontrolled

535 Eating (UE) and the general impulsivity-related variables stress reactivity, lack of self-control

536 and reward sensitivity. B) Model $4(\mathrm{n}=1197)$, a model that additionally includes the associations

537 between UE, general impulsivity-related variables, BMI and 6-month longitudinal change in

538 BMI (delta BMI) are added. Numeric values are standardized beta weights (all of them $\mathrm{p}<0.005$ ).

539 Dashed gray lines depict non-significant associations. PSS - perceived stress scale. UE -

540 Uncontrolled Eating. BMI - body mass index. Stress - stress reactivity. Reward - reward

541 sensitivity. Disinh. - disinhibition. Emot. Eating - emotional eating. Lack Persev. - lack of

542 perseverance. Sensat. seeking - sensation seeking. 
bioRxiv preprint doi: https://doi.org/10.1101/348821; this version posted September 8, 2020. The copyright holder for this preprint (which was not certified by peer review) is the author/funder, who has granted bioRxiv a license to display the preprint in perpetuity. It is made available under aCC-BY-NC-ND 4.0 International license.

A

Stress reactivity
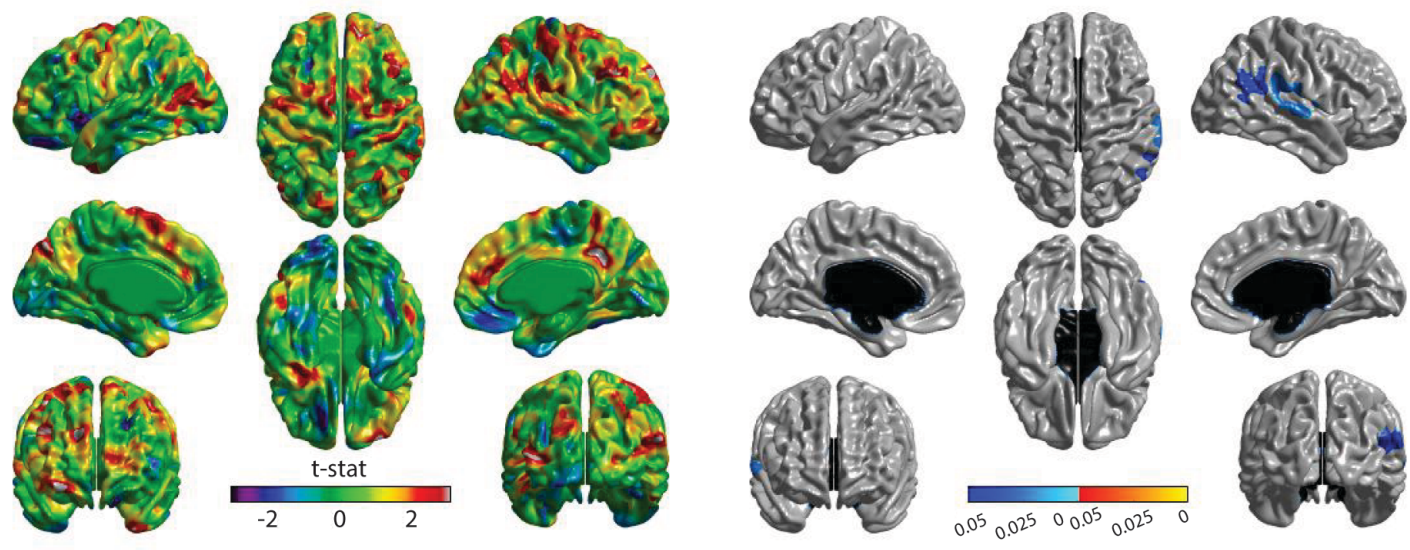

B
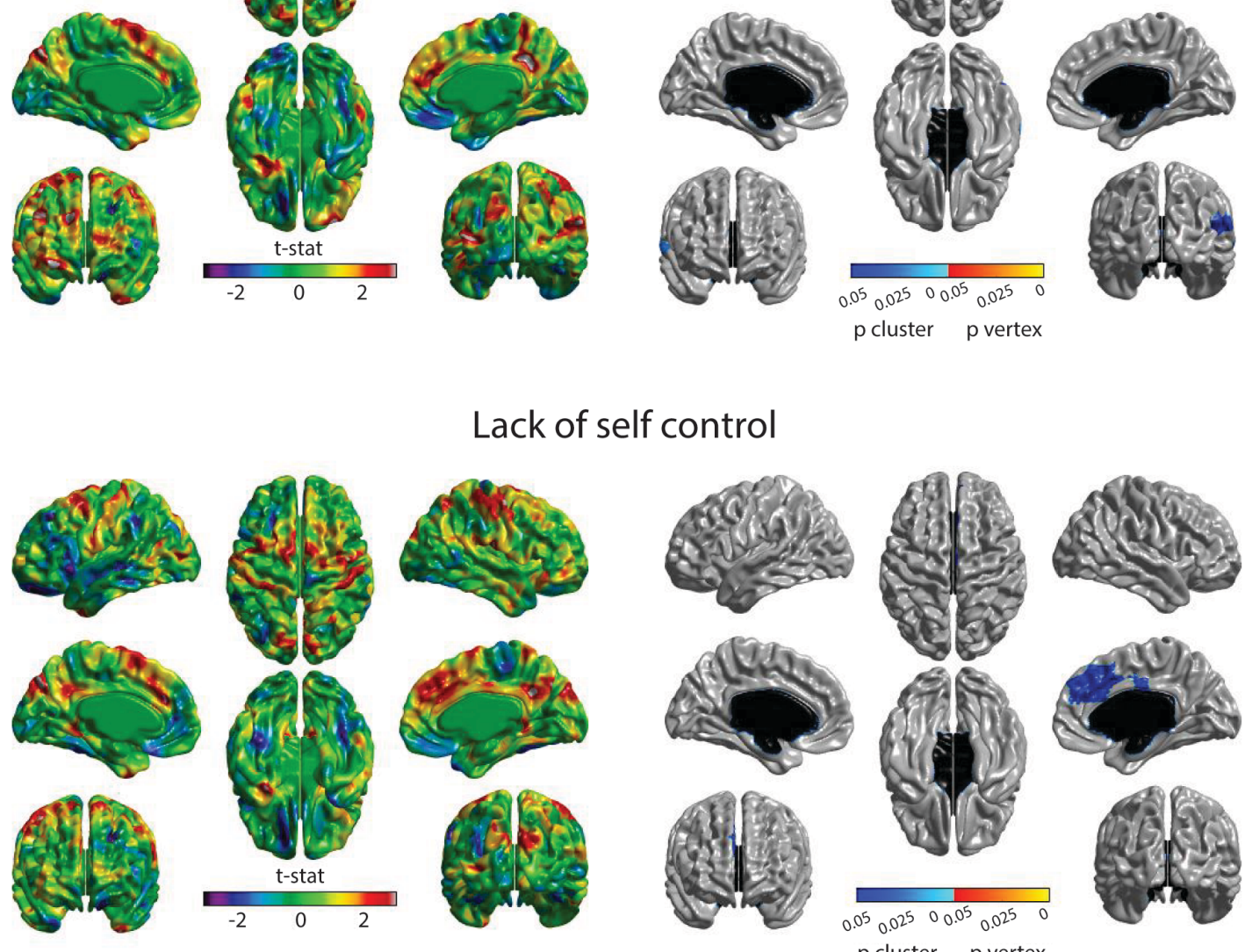

\section{Lack of self control}
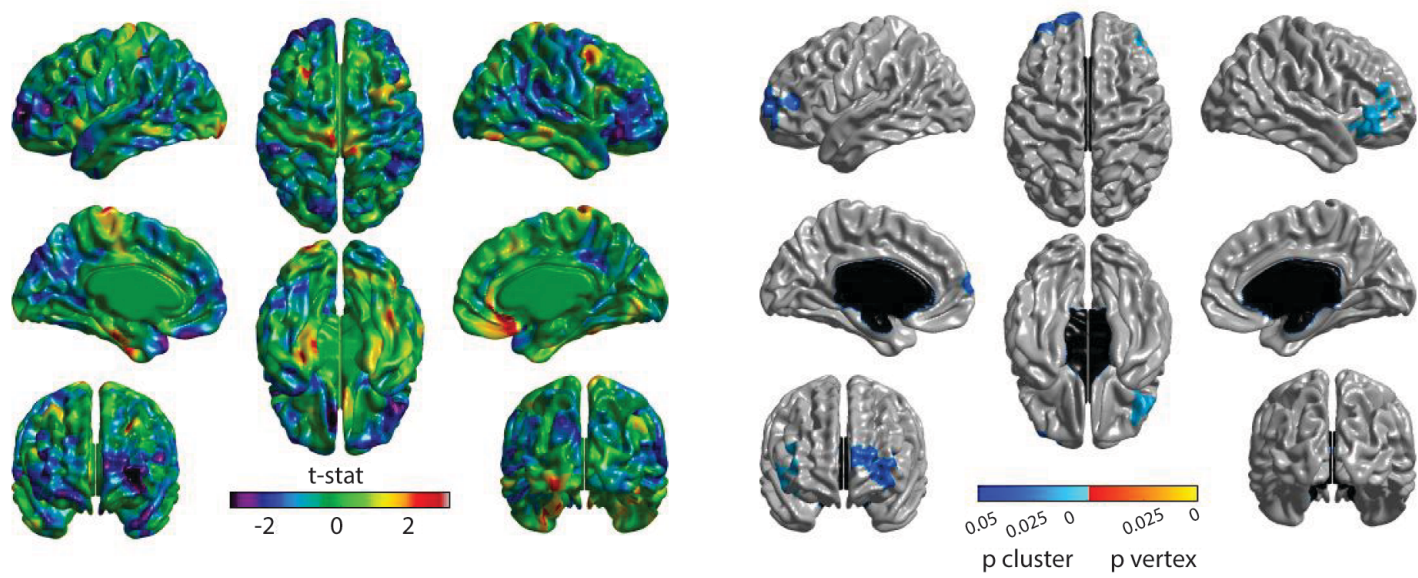

544 Figure 3 Correlations between latent factors of impulsivity and cortical thickness. 
$\underline{\text { Table }}$

Table 1. Descriptive statistics of the study variables. Numbers in parentheses indicate standard

547 deviation.

\begin{tabular}{|c|c|c|c|}
\hline Variables & $\begin{array}{r}\text { Full sample } \\
(\mathbf{n}=\mathbf{2 3 1 8})\end{array}$ & $\begin{array}{r}\text { Subsample with } \\
\text { longitudinal BMI } \\
(\mathrm{n}=1197)\end{array}$ & $\begin{array}{r}\text { Subsample with } \\
\text { MRI }(n=71)\end{array}$ \\
\hline Age & $19.34(4.35)$ & $19.40(4.07)$ & $18.24(0.80)$ \\
\hline \multirow[t]{2}{*}{ Sex } & 780 males & 347 males & 18 males \\
\hline & 1538 females & 830 females & 53 females \\
\hline Baseline (autumn) BMI (kg/m²) & $22.24(3.57)$ & $22.21(3.44)$ & $22.38(2.54)$ \\
\hline Follow-up (spring) BMI $\left(\mathrm{kg} / \mathrm{m}^{2}\right)$ & & $22.36(4.25)$ & $22.48(2.63)$ \\
\hline Change in weight in first year $(\mathrm{kg})$ & & $0.52(3.35)$ & $1.39(2.67)$ \\
\hline $\begin{array}{l}\text { Disinhibition (Three-Factor Eating } \\
\text { Questionnaire) }\end{array}$ & $6.38(3.34)$ & $6.38(3.42)$ & $6.24(3.47)$ \\
\hline $\begin{array}{l}\text { Emotional Eating (Dutch Eating } \\
\text { Behavior Questionnaire) }\end{array}$ & $2.45(0.74)$ & $2.42(0.77)$ & $2.36(0.70)$ \\
\hline Power of Food (PFS) & $12.42(3.65)$ & $12.47(3.84)$ & $12.40(3.72)$ \\
\hline $\begin{array}{l}\text { Lack of Perseverance (the UPPS } \\
\text { Impulsive Behavior Scale) }\end{array}$ & $1.91(0.45)$ & $1.91(0.47)$ & $1.97(0.49)$ \\
\hline $\begin{array}{l}\text { Sensation Seeking (the UPPS Impulsive } \\
\text { Behavior Scale) }\end{array}$ & $2.81(0.56)$ & $2.91(0.59)$ & $2.87(0.55)$ \\
\hline Rosenberg Self Esteem Scale & $29.85(4.96)$ & $29.66(5.30)$ & $28.92(5.86)$ \\
\hline Cohen's Perceived Stress Scale & $24.57(7.17)$ & $24.82(7.35)$ & $24.48(7.79)$ \\
\hline $\begin{array}{l}\text { Neuroticism (International Personality } \\
\text { Item Pool) }\end{array}$ & $11.63(2.93)$ & $11.59(3.17)$ & $11.69(3.08)$ \\
\hline $\begin{array}{l}\text { Agreeableness (International } \\
\text { Personality Item Pool) }\end{array}$ & $15.60(2.80)$ & $15.67(3.01)$ & $15.73(2.86)$ \\
\hline $\begin{array}{l}\text { Imagination (International Personality } \\
\text { Item Pool) }\end{array}$ & $15.15(2.75)$ & $15.16(2.90)$ & $15.73(2.86)$ \\
\hline $\begin{array}{l}\text { Conscientiousness (International } \\
\text { Personality Item Pool) }\end{array}$ & $13.97(2.93)$ & $13.98(3.18)$ & $13.46(3.13)$ \\
\hline $\begin{array}{l}\text { Extraversion (International Personality } \\
\text { Item Pool) }\end{array}$ & $12.04(3.48)$ & $11.86(3.66)$ & $11.77(4.13)$ \\
\hline
\end{tabular}

